\title{
IGSA-FA for Optimal Placement of FACTS Devices
}

\author{
Ramakanta Jena, Sarat Chandra Swain, Ritesh Dash
}

\begin{abstract}
With the globalization of power market by reducing the installation and operating cost of the power plant with profitable power flow controller leads to successful implementation of optimal power flow through optimal algorithms. Finding the solution of optimal load flow problem with non-linear equation such as Newton's equation is one of the possible solution. However, applying Newton's solution to OPF for finding convergence is a little bit tedious and time consuming affecting marginal losses by involving a number of inequalities present in the system. Transmission lines capacity and bus voltage limit are vital safety factors to carry out $O P F$ in any power system The system being operational in normal state is equipped with security measures in order to discern that it is capable of resisting contingencies devoid of any limit contravention. To ensure a consistent power system function, it is essential that the safety of the system is duly accounted for in
\end{abstract}

Keywords : About four key words or phrases in alphabetical order, separated by commas.

\section{INTRODUCTION}

.Voltage limit of the Bus and its capacity are the two most important parameters to carry out the Optimal Power Factor study in a IEEE standardized bus system. Due to the limitation of the traditional methd of optimization theory to solve non-linear equations , these are not directly applied to solve OPF. Again the convergence pf these equations depends upon the initial condition therefore feasibility is always uncertain about OPF. Therefore, a number of metaheuristic Optimisation techniques developed over the year to solve various optimal power flow problem with single and multidimensional constraints. Some of the most advanced population control algorithm surcharge differential evolution gravitational search algorithm genetic algorithm and differential evolution has been involved a lot of constants to solve the optimality of the on constraint characteristic equation. The flexible AC transmission devices are basically used in power system to cut down the flow of heavily loaded system to an optimized for and thereby increasing the ability of the transmission line to carry power to eat optimality label reducing the damage of power transmission line. Out of the number of devices such as controlled series compensator is

Revised Manuscript Received on December 30, 2019.

* Correspondence Author

Ramakanta Jena, Research Scholar, School of Electrical Engineering KIIT University, Bhubaneswar, Odisha, India. Email: rjenafel@gmail.com

Dr. Sarat Chandra Swain, Professor, School of Electrical Engineering, KIIT University, Bhubaneswar, Odisha, India.

Email: scs_132@rediffmail.com

Dr. Ritesh Dash*, Asso. Professor, Christian College of Engineering \& Technology, Bhilai, India. Email: rdasheee@gmail.com

(C) The Authors. Published by Blue Eyes Intelligence Engineering and Sciences Publication (BEIESP). This is an open access article under the CC BY-NC-ND license (http://creativecommons.org/licenses/by-nc-nd/4.0/) generally Android with a number of facility such as power flow control on communication line and damping out power oscillation between inter area decreasing and enhancing the transient stability. Therefore it is required to to design and office which can accommodate flexible AC transmission system and its characteristic like providing voltage stability to an optimal power flow solution. Different types of algorithms such as genetic algorithm and symbiotic organism search algorithm and oppositional head algorithm have been introduced by many researchers in the past year problem has been truly addressed based on its Diagnostic acid investigation method with the fact devices. The researcher are working throughout the world to develop more sophisticated power flow algorithm based on metaheuristic technique so that a natural Optimisation can be achieved with minimal constant. In Chapter optimal placement of thyristor controlled switch capacitor has been discussed along with application of gravitational search algorithm has been described to establish relationship between voltage and finding out the relationship between optimal power flow and Facts devices. The main advantage of the algorithm is that it is completely divided by Gravity constant and the efficiency of the system is enhanced with memoryless techniques. The performance of the proposed technique has been verified with IEEE 5 and 9 Bus system. The simulation result obtained from matlab programming has been verified with result described in the literature for stability analysis of optimal power flow algorithm

\section{TCSC MODELLING \& PROBLEM FORMULATION}

Thyristor controlled switch capacitor is one of the most versatile facts devices which encompasses capacitor shunted with controlled reactor. Hear the reactance is connected in series with the transmission line and the line impedance so adjust that it can adopt the Apparent power depending upon the loading condition light. the below figure shows the Great interconnection between Bus 1 and Bus2. The main objective of the optimal power flow is to control the flow fuel to the generation system for lowering the cost of production. The objective function with all constraints are incorporated here as in equation 4.1

Fuel Cost $=F c-\Sigma\left(a_{n}+b_{n} p_{g i}^{1}+c_{n} p_{g i}^{2}\right)$

From the point of view point of view TCSC is analysed equality and inequality constraints for finding out the optimal power flow in 5 bus and 9 bus system. Balancing Apparent power can be regarded as equality constraint real power and system voltage along with frequency can be regarded as inequality constraint.

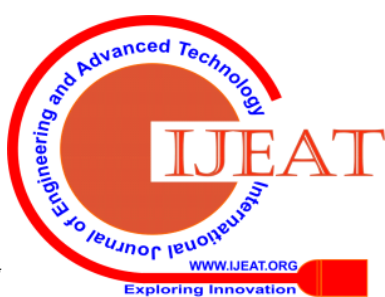


Finding out the factor involved in optimal power flow along with different it security constraint is the main objective of investigating the optimal option of TCSC. Loading factor can be made proportional by increasing the active and reactive power of the considered load. This method will change the loading factor from its base value to the maximum value without affecting the equality and inequality constraint. In order to calculate the best for the maximum limit of the boundary value has been taken into consideration for finding out maximum power flow limit. Amalgamation of loading factor and maximum flow of limit can lead to the calculation of Apparent power flow index. Therefore, apparent power flow index can be calculated as

Highest value of Apparent power index indicates that the considered bus for placement of fact devices is a weak bus. This index determines the voltage variance and cost function thereby calculating the optimal capacity of facts devices. Therefore it can be concluded that the two parameters must be kept small as possible.

The Main objective of optimal power flow problem is established relationship between the control variable parameter for minimising the objective function Subject to Equality and inequality constraint. Hence the optimization problem can be written as

$$
\begin{aligned}
& \text { Minimize } f(x, u)=0 \\
& \text { S.t } g(x, u)=0 \\
& \text { And } h(x, u)<0
\end{aligned}
$$

Here $\mathrm{f}$ represent the objective function to be minimised and $g$ and $h$ represents the equality and inequality constraints Associated the objective function for finding out the solution. All the state variables used in objective function are represented by X. similarly, the vector control variables are represented by u. Load bus parameter is same as The generator output power And the bus voltage of constrained and unconstrained parameters. Optimal placement of TCSC Satisfying optimal power flow Under normal and abnormal condition such as overvoltage and undervoltage condition has been discussed in this chapter. As already mentioned power balancing equation is considered in this case as equality constant Where as voltage magnitude, generator power, and reactance of TCSC are considered as inequality constraints. The detailed description of the above constraints are described in the next paragraph. The optimal location of facts devices in a transmission line depends upon its objective function and the parameters considered for calculation of optimal power flow. in order to calculate voltage variance and cost function in this case Firefly algorithm is implemented and describe in detail along with facts devices (TCSC). Assurance to get optimal voltage stability is not guaranteed in this approach although different voltage limit has been taken into consideration for calculating the optimality. therefore additional voltage level constant has been taken into optimal power flow problem. This approach restricts the optimal solution to reach its maximum value though critical condition even if voltage collapse situation appears during the optimality testing. The voltage variation constraints can be formulated with reference to maximum permissible value voltage different Bus to that of threshold voltage of the system.

$v_{d \max } \leq v_{d \text { limit }}$

And

Whereas
$v_{d}=\sum_{i=1}^{N}\left(v_{n}-v_{i}\right)^{2}$

Here Vd represents the maximum allowable error between Bus voltage and its transient over voltages. $\mathrm{Vn}$ is the Bus voltage under normal condition and Vi represents the over voltage at ith bus during transient operation condition. The main objective of extra voltage limit is to protect the system from voltage collapse and provides extra system stability. From the above statement it can be concluded that the initial investment in FACTS devices is much more higher than the transmission lines however in a long run its payback period is much smaller as compared to any other static device for providing voltage compensation. Optimal installation of FACTS devices such as TCSC will also reduce the over cost of installation for providing the same short of protection and voltage compensation.

\section{GRAVITATIONAL SEARCH ALGORITHM}

This section describes about the optimization of TCSC controller in its deciding the optimal place for providing location which involves multi objective optimization algorithm to solve optimal power flow and voltage stability were presented in this section. Again as said earlier that the optimal location of the TCSC depends upon the API Index therefore evaluation of effectiveness of the said algorithm is an essential part. Two methods are depicted to establish the relationship between the two variables such as finding the security isses involved in optimal placement of OPF and second method is of cost minimizing by for TCSC along with enhancing the voltage stability.

Here hybrid technique is applied to find the optimal place of the TCSC by combining two optomisation techniques such as Gravity Search Algorithm and FA. The process of finding the optimal location for placement of the TCSC involves two step, in the First step normal Newton-Raphson method is applied to the system BUS for finding the initial power flow, Bus voltage, Real and Reactive power at each Bus. In the second step a fault is introduced at a BUS thereby making the system instable. Here API of all the Buses were calculated and maximum API corresponds to the Bus will be identified as weak Bus of the system. The OPF loop will be automatically terminated after achieving the maximum API however if the convergence will not appear with the selected constraints then the boundary value of the constraints will be increased to a new value corresponding to the system equality and inequality constraints so as to sure the Optimal Power Flow. In case any violation exist the OPF loop will be repeated again and again till maximum API is achieved to that corresponding Bus. After identifying the weakest Bus Firefly algorithm will be applied to find out the best capacity of TCSC this will recover the normal operating condition and thereby enhancing the voltage stability. The differential voltage will be verified with that of the transmission line reactance whose equivalent in the form of TCSC was placed in the transmission lines. Voltage variation around the maximum transient limit will be carried out to check wheather optimal place and location has been found out or not. The entire system was investigated with IEEE 5 Bus and 9 Bus system with an objective of voltage stability and OPF condition.

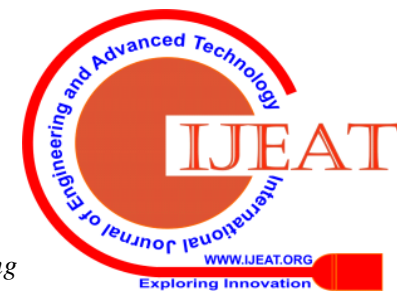


Gravitational search algorithm was introduced in the year 2009 to solve some Optimisation problem with constraint and unconstraint parameters. The basic structure of gravitational search algorithm based on Law of Gravity and its interaction with mass. The main aim of the gravitational search algorithm age to find searchable agent which interact among themselves show the gravitational forces. here is searchable object is considered to be a mass and there performance is measured by their mass. Movement of heavier mass is carried out under the action of gravity and thereby moving the entire function towards heavier mass under the action of gravity.

The slow convergence of Guarantees that the convergence of the system is as per the planned. All the masses present in the equation obey The law of gravity.

$\mathrm{F}=\mathrm{G}(\mathrm{M} 1 \mathrm{M} 2 / \mathrm{R} 2)$

here $\mathrm{F}$ represent Gravitational force and $\mathrm{G}$ represent gravitational constant . M1 and M2 represent mass of the two different objects and R2 represent distance between the two object. According to Newton's first law the gravitational force depends upon Mass of the object and inversely proportional to the square of distance between them. Where add second law of Newton's said that acceleration depends upon applied force upon mass. In gravitational search algorithm there are four parameters acceleration initial mass, active gravitational mass and passive gravitational mass which controls the entire objective function and their behaviour towards optimal feasibility solution. The feasibility of the solution depends upon inertial mass and active gravitational mass. This also relates to the fitness value of the feasibility solution. The entire algorithm is to be search it initial mass and gravitational mass where which mass represent a particular solution. The heaviest mass present in the solution represent the most optimal solution. Different steps involved in gravitational search algorithm are as follows.

- Agent Initialization

- Fitness assign and finding the best fitness value

- Calculation of gravitational constant

- Agent mass calculation

- Calculating the acceleration of agent

- Velocity of Agent

Position of n number of object was initializing randomly with the dimension and agent. Which can be written as $x_{i}=\left(x_{i}^{\mathbb{n}} \ldots \ldots x_{i}^{d} \ldots \ldots x_{i}^{n} \ldots \ldots\right)$, for $\mathrm{i}=1,2 \ldots \mathrm{N}$

For minimising or maximizing a function The best value is always find out by finding the best and worst fitness function for each objective that is mass.

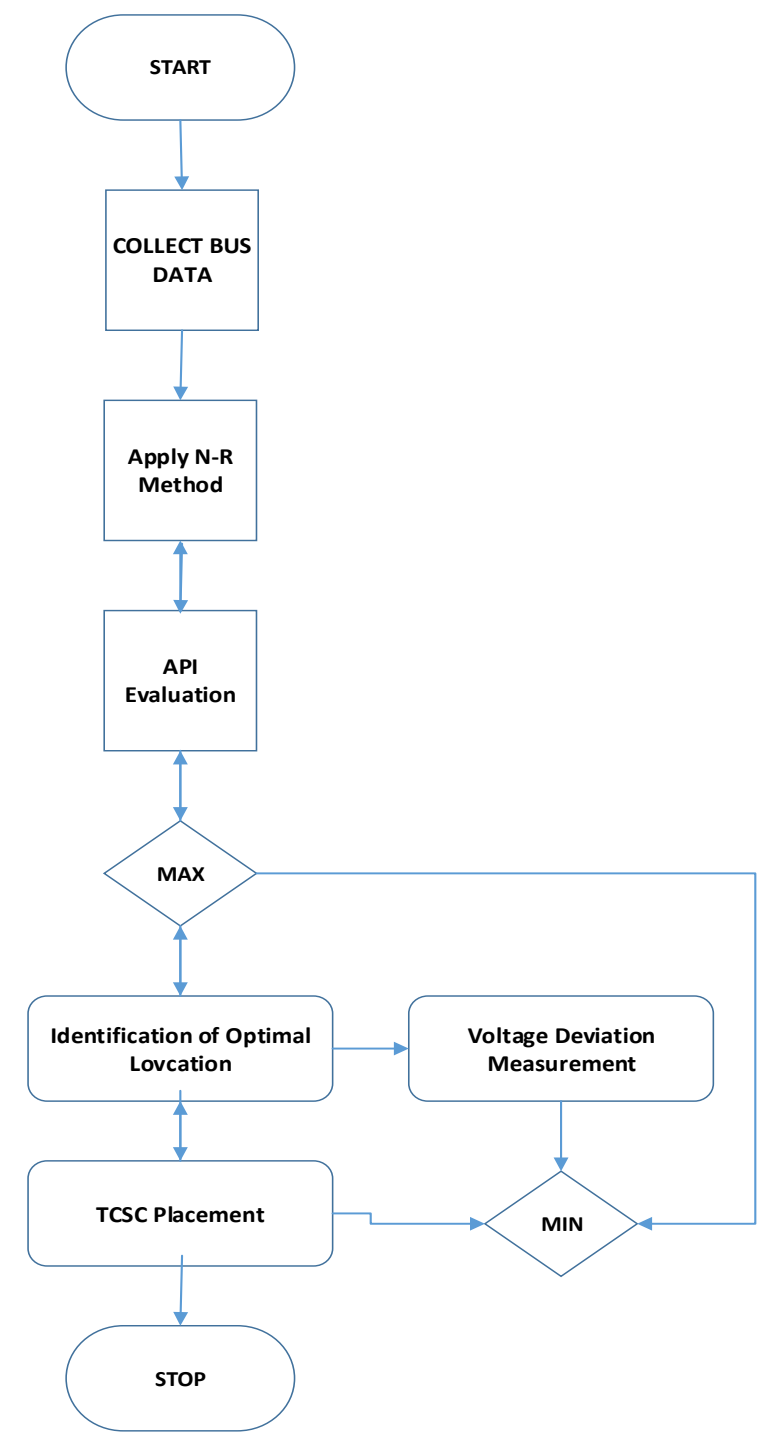

Fig.1 Flow Chart showing different steps involved in optimal placement of TCSC

\section{RESULT ANALYSIS}

In the present chapter, hybrid techniques such as IGSA along with FA is employed to suitably place the TCSC in its optimal position with minimizing the cost of production such as fuel cost and thereby decreasing the losses present in the system. The entire system was implemented in MATLAB 2019b with algorithm and Simulink model and was tested with IEEE 5 bus and 9 Bus system. As already discussed in the earlier chapter that newton- Raphson method is applied to calculate the different types of power loss and power flow occurring in the system i.e. with TCSC and without TCSC. The output of the method is compared with all other hybrid optimization techniques such as ANFIS, Fuzzy as already discussed in the previous chapter. All the Bus data, line data and all other required parameters that are required for power flow analysis are used in this chapter is same as that of the parameters used in the previous chapter. The detailed analysis which was carried out in this chapter is as follows.

i.Power flow analysis was carried out with

Newton-Raphson method at base load condition. ii. Varying the loading condition in the system subject o OPF. 
iii. Finding the best place for OPF solution to suitable place the TCSC at its optimal place in the IEEE-5 Bus and 9 Bus system.

Case-1: Power Flow Analysis with IEEE-5 Bus system

In this section application of the proposed system to IEEE-5 Bus has been described. The described system is verified along with the different types of stability condition. Here it is conditionally applied to test the performance of system for voltage stability, power losses that are occurring in the power system. The proposed system has been predicted to analyze the total active power loss occurring in the system along with the generation cost and effectiveness of placing TCSC in the Proposed IEEE-5 Bus system. The given system was analyzed both under uncompensated and compensated condition.

From the Matlab Simulink result it can be observed that the real power flow between bus no. 3 and bus no. 4 increases beyond the normal power flow limits. Hence it is not recommended to operate the system under this condition again it is also violating the security issues associated with the transmission line system. Optimal power flow solution achieved in this case upon overloading the system does make it suitable to operate the system under security condition. A system is said to be highly secured only when it is optimized with over loading condition. From the previous chapter and the current simulation result shows that Optimum condition for placement of TCSC can be achieved with Fuzzy and ANFIS and also it is found that the suitable place for placement of TCSC is in between Bus No. 4 and 5, however reduction in the power loss, lower cost of generation and line flow from TCSC point of can be effectively achieved by optimizing the system with IGSA along with FA. From the result it can also be understood that the A\&T losses occurring in the system has also been reduced by $67 \%$ as compared to other techniques presented in the system. Table No. 4.1 shows the detail power flow analysis of the proposed system along with other techniques both under the compensated condition and uncompensated condition.

Table.1 Optimal Power Flow and Reactive Power Profile for IEEE-5 Bus

\begin{tabular}{|c|c|c|c|c|}
\hline $\begin{array}{c}\text { Sl. } \\
\text { No. }\end{array}$ & $\begin{array}{c}\text { Details of } \\
\text { Reactive } \\
\text { Power }\end{array}$ & $\begin{array}{c}\text { Normal } \\
\text { Situation }\end{array}$ & Loaded & IGSA-FA \\
\hline 01 & $\begin{array}{c}\text { Total } \\
\text { Reactive } \\
\text { Power }\end{array}$ & 23.43 & 18.63 & 21.16 \\
\hline 02. & $\begin{array}{c}\text { Total } \\
\text { Reactive } \\
\text { Power Loss }\end{array}$ & 2.72 & 2.53 & 2.36 \\
\hline 03. & $\begin{array}{c}\text { Reactive } \\
\text { Power } \\
\text { Demanded }\end{array}$ & 17.26 & 16.85 & 16.78 \\
\hline
\end{tabular}

Voltage at different Buses is also considered in this chapter or project work as a part of the security issues therefore voltage differences at different buses both during Normal Loaded Condition and IGSA-FA loaded condition is shown in table 4.3

Table.2 Optimal Power Flow and Bus Voltage Variation for IEEE-5 Bus

\begin{tabular}{|c|c|c|c|}
\hline $\begin{array}{c}\text { Sl. } \\
\text { No. }\end{array}$ & $\begin{array}{c}\text { Bus } \\
\text { No. }\end{array}$ & $\begin{array}{c}\text { Normal Condition } \\
\text { with TCSC }\end{array}$ & $\begin{array}{c}\text { IGSA-FA Condition } \\
\text { with TCSC }\end{array}$ \\
\hline 01. & 1 & 1.06 & 1.05 \\
\hline 02. & 2 & 1.06 & 1.04 \\
\hline
\end{tabular}

\begin{tabular}{|c|c|c|c|}
\hline 03. & 3 & 1.07 & 1.06 \\
\hline 04. & 4 & 0.9 & 1.0 \\
\hline 05. & 5 & 0.9 & 1.0 \\
\hline 06. & 6 & 0.9 & 1.0 \\
\hline
\end{tabular}

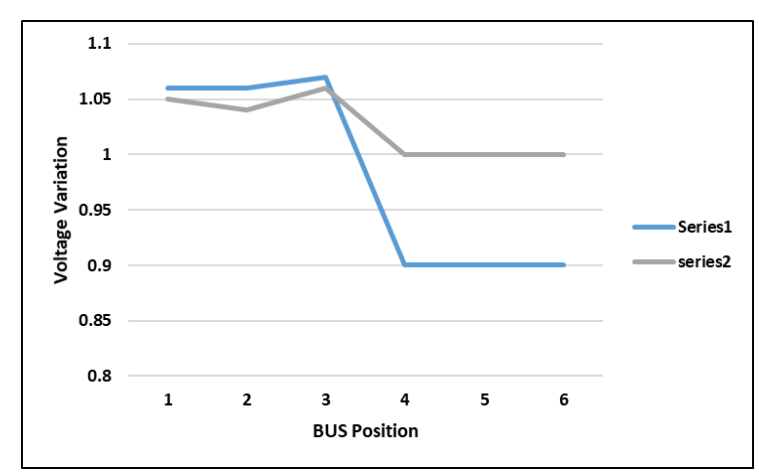

Figure. 2 IEEE-6 Bus Voltage Variation under Normal Operating Condition and IGSA-FA Condition

Individual line-loss is as follows

Table.3 Line Losses Occurring at Different Buses for an IEEE-6 Bus System

\begin{tabular}{|c|c|c|}
\hline Sl. No. & Line Code & Line Los \\
\hline 1 & $1->2$ & $(0.014105)+\mathrm{j}(0.024308)$ \\
\hline 2 & $1->3$ & $(0.011920)+\mathrm{j}(0.018555)$ \\
\hline 3 & $2->3$ & $(0.003515)+\mathrm{j}(0.032376)$ \\
\hline 4 & $2->4$ & $(0.004413)+\mathrm{j}(0.029656)$ \\
\hline 5 & $2->5$ & $(0.011252)+\mathrm{j}(0.001758)$ \\
\hline 6 & $3->4$ & $(0.000356)+\mathrm{j}(0.019898)$ \\
\hline 7 & $4->5$ & $(0.000307)+\mathrm{j}(0.051176)$ \\
\hline
\end{tabular}

\section{CONCLUSION}

As clearly stated at the beginning of the chapter, the recent project work shown in this chapter emphasizes about the optimal placement of TCSC controller for achieving voltage stability during both light load condition and heavy load condition with cost reduction and power loss minimization. Standard IEEE bench mark system i.e.5 Bus. The decreasing power flow security from light load condition to heavy load condition is restored with the help of suitably placing the TCSC in the studied IEEE-Bus system.

\section{REFERENCES}

1. Box, G.E.; Jenkins, G.M.; Reinsel, G.C.; Ljung, G.M. Time Series Analysis: Forecasting and Control; John Wiley \& Sons: Hoboken, NJ, USA, 2015.

2. Chatfield, C. The Analysis of Time Series: An Introduction; CRC Press: Boca Raton, FL, USA, 2016.

3. Marelli, D.; You, K.; Fu, M. Identification of ARMA models using intermittent and quantized output observations. Automatica 2013, 49, 360-369. [CrossRef]

4. Zhang, G.P. Time series forecasting using a hybrid ARIMA and neural network model. Neurocomputing 2003, 50, 159-175. [CrossRef]

5. Bigovi'c, M. Demand forecasting within Montenegrin tourism using Box-Jenkins methodology for seasonal ARIMA models. Tour. Hosp. Manag. 2012, 18, 1-18.

Published By:

Blue Eyes Intelligence Engineering \& Sciences Publication 
6. Kova 'ci'c, Z.J. AnalizaVremenskihSerija; EkonomskiFakultet Beograd: Beograd, Serbia, 1995. 47. Haykin, S. Network, Neural: A comprehensive foundation. Neural Netw. 2004, 2, 41.

7. Ritesh Dash, Sarat Chandra Swain, Effective Power quality improvement using Dynamic Activate compensation system with Renewable grid interfaced sources, Ain Shams Engineering Journal, Volume 9, Issue 4, 2018, Pages 2897-2905,

\section{AUTHORS PROFILE}

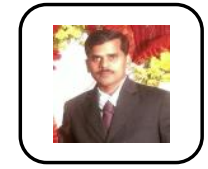

Ramakanta Jena is working as Research Scholar at School of Electrical Engineering. His Research Area is Sustainable Energy, especially optimization and its stability analysis,

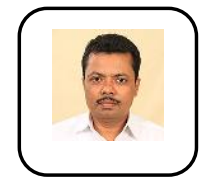

Dr. Sarat Chandra Swain presently working as a Professor cum Associate Dean in School of Electrical Engineering at KIIT University. He has a research experience of over 20 years and a pioneer in the field of Artificial Intelligence, FACTS and Electrical Drives. He both in International Journal and Conference. has published more than 100 numbers of research papers

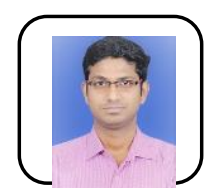

Dr. Ritesh Dash, presently working as Associate Professor at Christian College of Engineering \& Technology, Bhilai. He has a research experience of over 8 years and has sound knowledge in the field of Artificial Intelligence, FACTS and Machine learning. He has published more than 70 numbers of research papers both in International Journal and Conference. He has also served the Govt. of India as a Design Engineer, Electrical at WAPCOS Ltd. A Central PSU under Ministry of Water Resources \& Ganga Rejuvenation. He is associated with Many International Bodies such as IEEE, Indian Science Congress, The Institution of Engineers, 